\title{
Structure and Catalysis of NiOOH: Recent Advances on Atomic
}

\section{Simulation}

Ye-Fei Li*, Ji-Li Li, and Zhi-Pan Liu*

Collaborative Innovation Center of Chemistry for Energy Material, Key Laboratory of Computational Physical Science (Ministry of Education), Shanghai Key Laboratory of Molecular Catalysis and Innovative Materials, Department of Chemistry, Fudan University, Shanghai 200433, China

Email: yefeil@,fudan.edu.cn; zpliu@,fudan.edu.cn

\begin{tabular}{cc}
\hline Table S1. Correspondence between two sets of Miller-Bravais indices \\
\hline conventional & Carter's \\
\hline$(10 \overline{1} \overline{2})$ & $(10 \overline{1} 0)$ \\
$(\overline{1} 101)$ & $(\overline{1} 100)$ \\
$(01 \overline{1} \overline{1})$ & $(01 \overline{1} 0)$ \\
$(01 \overline{1} 2)$ & $(01 \overline{1} 1)$ \\
$(01 \overline{1} 5)$ & $(01 \overline{1} 2)$ \\
$(11 \overline{2} 3)$ & $(11 \overline{2} 0)$ \\
$(\overline{1} 2 \overline{1} 3)$ & $(\overline{1} 2 \overline{1} 1)$ \\
$(2 \overline{1} \overline{1} 3)$ & $(2 \overline{1} \overline{1} 0)$ \\
\hline
\end{tabular}

